less consistent as the subjects do not always return to the hospital as requested.

Blood samples were also taken from each of the subjects at the beginning of the quarantine period, and they will be taken again this week. These will be tested for the presence of antibodies against the coronavirus, which will show whether the subjects have been exposed to the virus and have mounted a successful defence. Lim is not prepared to confirm how many of the study's subjects have now developed symptoms of the disease.

Data from these samples, as well as from patients suffering from SARS, will offer clues to some of the key biological questions, says Lim, including when the virus starts to show up in bodily fluids, where in the body it comes from, and whether there are symptomless carriers. "At the moment we don't know enough about the route of transmission of the virus," Lim says. "There is just not enough material yet for investigation."

The study has also analysed hundreds of samples taken from surfaces in the Amoy Gardens apartments, such as toilet seats and door handles, some of which have tested positive for SARS in PCR tests.

In the next few weeks, Lim's group will also begin a study on people from outside Amoy Gardens who have had contact with the disease but have yet to display symptoms.

Other researchers involved in the global network established by the World Health Organization (WHO) in response to the SARS outbreak confirm that it will be several weeks, or even months, before they will

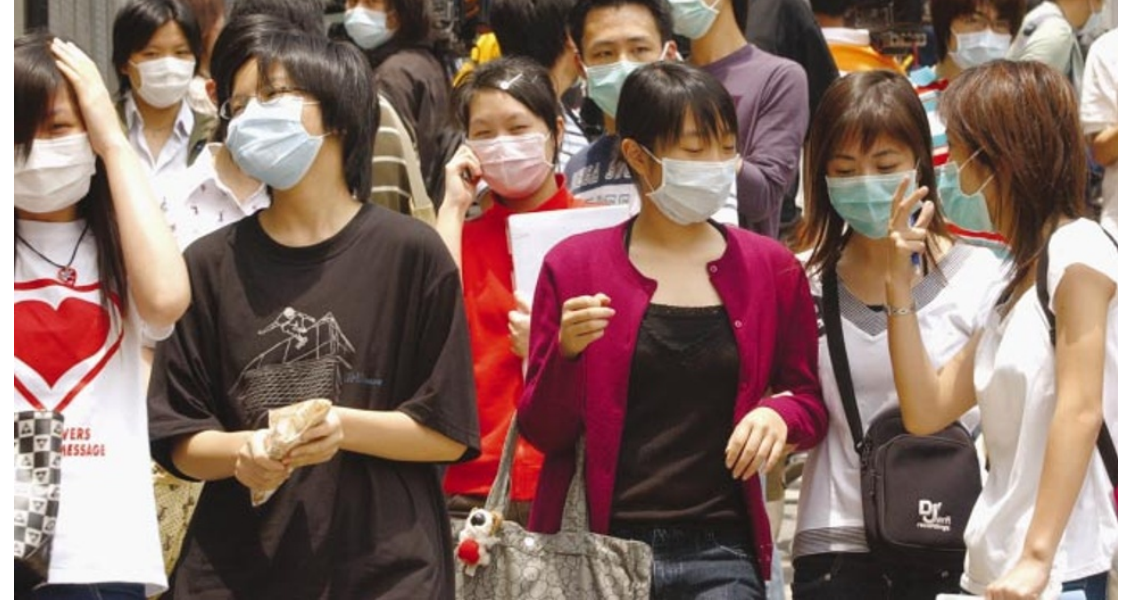

Covered up: students in Kowloon protect themselves following the area's outbreak of SARS.

have enough data to make even the broadest of predictions about the disease's spread.

Samples from patients from countries where SARS infection has been significant, including Hong Kong, Vietnam and Singapore, are also being tested regularly to determine how best to track the virus during the course of the disease. Results of PCR analysis show that stool samples test positive for viral particles for up to 30 days, by which time symptoms have often disappeared. The particles seem to be infective for up to ten days - once isolated they can infect normal cells in culture - but it has not yet been established whether they stay infective after that.

Tracking populations of sick patients and healthy people who have been in contact with them is a powerful method for trying to understand the progress of SARS, but many details can only be investigated in animal models. A monkey model based on macaques, which has just been developed at Erasmus University in Rotterdam, the Netherlands, is now also being used at the US Centers for Disease Control and Prevention in an attempt to understand factors such as how quickly symptoms appear after infection.

But with primates so expensive and awkward to work with, labs in the WHO network are trying to develop models for SARS in more convenient animals such as mice. "We'll need several months to get a suitable small animal model," says Christian Drosten, of the Bernhard Nocht Institute for Tropical Medicine in Hamburg, Germany.

The WHO, meanwhile, has already issued strict travel guidelines, assuming the worst. "We don't have the luxury of seeing what happens and then acting," says David Heymann, the WHO's executive director for communicable diseases.

i www.who.int/csr/sars/travel

\title{
Critics slam treatment for SARS as ineffective and perhaps dangerous
}

Health authorities in Hong Kong are coming under intense criticism over their use of an antiviral drug combined with anti-inflammatory steroids to treat patients with severe acute respiratory syndrome (SARS).

Critics of the treatment say that there are few indications that the combination of steroids and the antiviral drug ribavirin is effective. And autopsies revealing damage to organs other than the lungs have raised the question of

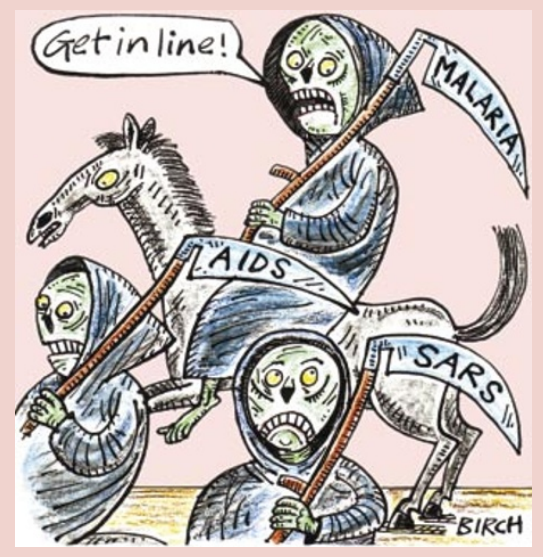

whether the drugs are partly to blame.

"There has been concern in Hong Kong about the efficacy of the treatment, and doctors there are calling for a re-examination of the protocol amid concerns that the prolonged use of highdose ribavirin may be causing or exacerbating multi-organ failure," says Gurinder Shahi, a physician and chairman of the Singapore-based biotechnology company BioEnterprise Asia.

Many in Hong Kong say that the necessary clinical tests have not been done, and that it is therefore too early to judge. "There is no clear evidence either way right now," says Ka-fai To, a pathologist at the Chinese University of Hong Kong whose autopsies of SARS victims found evidence of damage to the lymph nodes and spleen. "These findings could be side effects of the drug, but they could also be the effect of the virus or even an immune response to the virus," she says.

In Hong Kong, which has the largest number of SARS cases and deaths outside mainland China, all patients receive the combination treatment, says John Tam, director of virology at the Prince of Wales Hospital in Hong Kong. The combination is also being used in China's
Guangdong Province, Singapore and Toronto. Although ribavirin is known to be toxic in some patients, it has been prescribed because of its effectiveness against respiratory syncytial virus. This virus is similar to the metapneumovirus that was first isolated in Hong Kong and was originally thought to be the cause of SARS, says Masato Tashiro of the National Institute of Infectious Diseases in Tokyo.

But the drug's efficacy against SARS, which is now thought to be caused by a new strain of coronavirus, is unknown. "Increasing numbers of patients show no improvement," says Tam. In vitro tests are also negative, according to Tashiro.

But in the absence of any obvious alternative, health authorities in Hong Kong are sticking with the therapy. In a speech in Kuala Lumpur on 26 April, the health secretary Carrie Yau Tsang Ka-lai said that $80 \%$ of patients seem to respond favourably to the treatment.

But US officials have rejected ribavirin as a treatment for SARS. At a press briefing on 22 April, Julie Gerberding, director of the Centers for Disease Control and Prevention, said: "There doesn't seem to be any activity of ribavirin against this particular coronavirus." David Cyranoski 